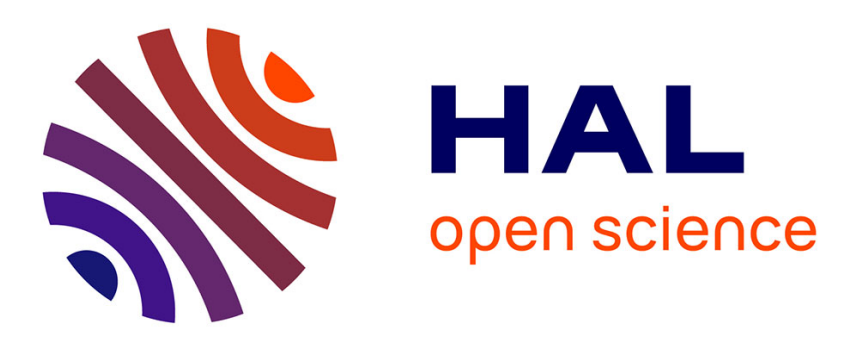

\title{
Self-regulating processes-based modeling for arrhythmia characterization
}

Antoine Echelard, Jacques Lévy Véhel

\section{To cite this version:}

Antoine Echelard, Jacques Lévy Véhel. Self-regulating processes-based modeling for arrhythmia characterization. Imaging and Signal Processing in Health Care and Technology, May 2012, Baltimore, United States. hal-00670064

\section{HAL Id: hal-00670064 \\ https://hal.inria.fr/hal-00670064}

Submitted on 14 Feb 2012

HAL is a multi-disciplinary open access archive for the deposit and dissemination of scientific research documents, whether they are published or not. The documents may come from teaching and research institutions in France or abroad, or from public or private research centers.
L'archive ouverte pluridisciplinaire HAL, est destinée au dépôt et à la diffusion de documents scientifiques de niveau recherche, publiés ou non, émanant des établissements d'enseignement et de recherche français ou étrangers, des laboratoires publics ou privés. 


\section{SELF-REGULATING PROCESSES-BASED MODELING FOR ARRHYTHMIA CHARACTERIZATION}

\author{
Antoine Echelard \\ Regularity Team \\ Inria \\ Ecole Centrale Paris - Grande Voie des Vignes \\ 92295 Châtenay-Malabry Cedex, France \\ email: antoine.echelard@gmail.com
}

\begin{abstract}
We propose a new model for RR interval records based on the empirical finding that the amplitude of RR records is negatively correlated with its regularity. We develop the mathematics needed to define rigorously this model, as well as the statistical tools necessary for estimating the parameters of the model on sampled data. We use this to analyze finely the impact of ventricular extrasystole of RR records, and to assess the efficiency of certain treatments on this pathology.
\end{abstract}

\section{KEY WORDS}

Arrhythmia, ECG, RR intervals, local regularity, multifractal processes, self-regulating processes

\section{Introduction}

Arrhythmiais a common condition that has many different causes and comes in various types. It can be of no or few consequences, or have dramatic effects. We will focus in our experimental study on ventricular extrasystole, although our methods should be applicable to other kinds of arrhythmia as well.

Arrhythmia may be detected from ECG, or from RR intervals extracted from ECG. A crucial concept related to arrhythmia is that of heart-rate variability. Indeed, the heart beats in an intrinsically variable way, due both to external events and inner regulation mechanisms. In view of the fact that ECG or RR interval records are extremely irregular, numerous papers have studied heart-rate variability from the point of view of fractal or multifractal analysis in recent years. See, among many others, [4, 5, 6, 8, 11]. All conclude that the studied signals (ECG or RR intervals) are indeed multifractal, and that pathologies are always associated with a reduction in multifractality, typically in the sense of a larger mean exponent and a narrower multifractal spectrum. In other words, a healthy heart is irregular, and, moreover, "irreluglarly" irregular, in the sense that its irregularity varies strongly in time. In addition to works dealing with multifractal analysis and heart rate variability in general, there is a number of studies dealing more specifically with the applications of multifractals to studying arrhythmia: see [2] (which also uses a robust fractal dimension

\author{
Jacques Lévy Véhel \\ Regularity Team \\ Inria \\ Ecole Centrale Paris - Grande Voie des Vignes \\ 92295 Châtenay-Malabry Cedex, France \\ email: jacques.levy-vehel@inria.fr
}

known as the regularization dimension [10]), [9, 12, 7, 13].

Multifractal analysis provides a statistical information describing the distribution of local regularity across the signal. We follows here a related but different approach: we track the local regularity of RR intervals at each point, and use this information to characterize the signal. More precisely, we provide empirical evidence that RR records display an intriguing feature: the time evolution of the signal is negatively correlated with the one of its regularity. In other words, at times where the RR signal is larger, it is also more irregular, and vice-versa. We call such a property the self-regulating property. While it is not easy at this stage to elucidate precisely the mechanisms leading to it, the idea that RR intervals are self-regulating should not come as a surprise: indeed, heartbeats are the results of complex interactions of several competing mechanisms that continuously corrects its rate in response to external and internal factors. This translates, in mathematical terms, into a process which, at each time, adjusts its variations (and thus its regularity) according to its current state (i.e. its amplitude). In order to turn this empirical finding into a useful method for analyzing, e.g., arrhythmia, we shall develop several ingredients:

1. a rigorous but tractable mathematical model for selfregulating processes,

2. a way to calibrate such a model on real data, i.e. to estimate the parameters of the model valid for a specific population,

3. and finally an analysis of how a given condition will perturb the self-regulation and how it may be statistically assessed from numerical estimations.

We proceed to detail these three points in the remaining of this work. We will then apply our tools to the study of ventricular extrasystole, and to the automatic assessment of the efficiency of three treatments, namely encainide, flecainide and moracizine, from RR records. 

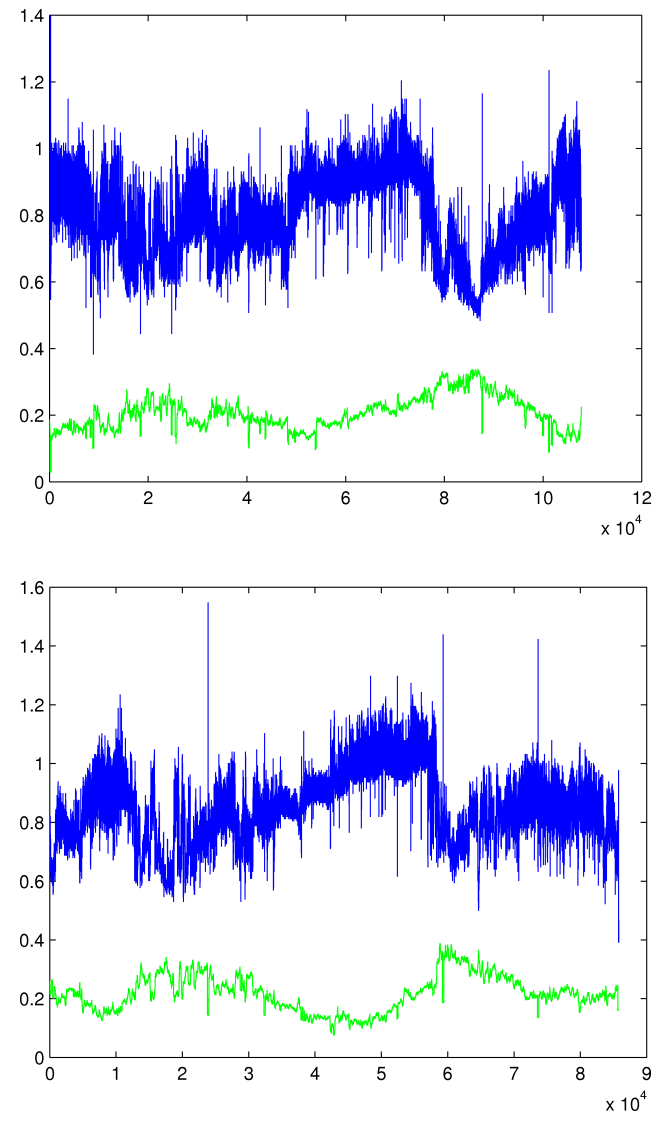

Figure 1. RR-Intervals (top graphs) and estimated pointwise regularity (bottom graphs)

\section{Empirical evidence of the self-regulating property of $R R$ intervals}

We show on figure 1 two typical 24 hours records of RR intervals, along with their estimated regularity (we have not yet stated precisely how we measure regularity, see 3.2 for a mathematical definition). As one can see, there is a strong (negative) correlation between the signal and its regularity. This calls for a new mathematical model, that would be able to account for this property and would allow a fine analysis of RR signals.

\section{The model}

\subsection{Definition of the self-regulating midpoint displace- ment process}

We wish to build a process whose local regularity is a function of the amplitude at each point. To make this precise,

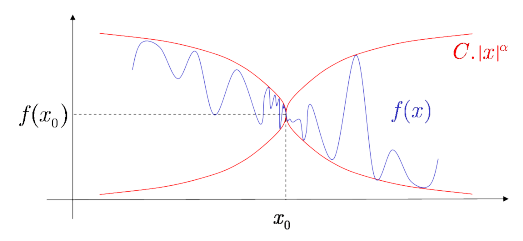

Figure 2. A function and its pointwise Hölder envelop

we first need a mathematical definition of local regularity. We will use the one at the basis of mulitfractal analysis, i.e. the pointwise Hölder exponent. Its definition in the case of interest to us goes as follows: let $f$ be a continuous but nowhere differentiable function from $\mathbb{R}$ to $\mathbb{R}$, and let $x_{0} \in \mathbb{R}$.

Definition 3.1. $f$ is said to belong the pointwise Hölder space $C^{\alpha}\left(x_{0}\right)$ if there exists $\varepsilon>0$ and $C \in \mathbb{R}$ such that, for all $t$ with $|t|<\varepsilon$ :

$$
\left|f\left(x_{0}+t\right)-f\left(x_{0}\right)\right| \leq C|t|^{\alpha}
$$

See figure 2 for a graphical illustration of this definition.

Definition 3.2. The pointwise Hölder exponent of $f$ at $x_{0}$ is the number:

$$
\alpha_{f}\left(x_{0}\right)=\sup \left\{\alpha: f \in C^{\alpha}\left(x_{0}\right)\right\}
$$

with the convention : $\sup (\emptyset)=0$.

Since the Hölder exponent exists at all points, we may define the Hölder function of $f$, denoted $\alpha_{f}$, which measures the regularity of $f$ and its evolution across time. If, instead of a function $f$, one deals with a stochastic process $X$, the Hölder exponent is defined in the same way. The function $\alpha_{X}$ is of course in this case itself a stochastic process.

We will say that a process $X$ is self-regulating if there exists a differentiable deterministic function $g$ such that, almost surely, for all times $t, \alpha_{X}(t)=g(X(t))$. This amounts to stating that, at all times, the regularity of the process is governed by the value of its amplitude, through the map $g$. A construction of a self-regulating process was first proposed in [3]. Here we will build a different process by modifying the celebrated "midpoint displacement" method of P. Lévy for constructing Brownian motion. We now briefly describe this construction.

Define the "triangle function"

$$
\varphi(t)=\left\{\begin{array}{lcc}
2 t & \text { for } & t \in[0,1 / 2] \\
1-2 t & \text { for } & t \in[1 / 2,1] \\
0 & \text { otherwise } &
\end{array}\right.
$$

and its dilated-translated versions $\varphi_{j k}(t)=\varphi\left(2^{j} t-k\right)$ (see figure 3 ). Our construction is an iterative one: 


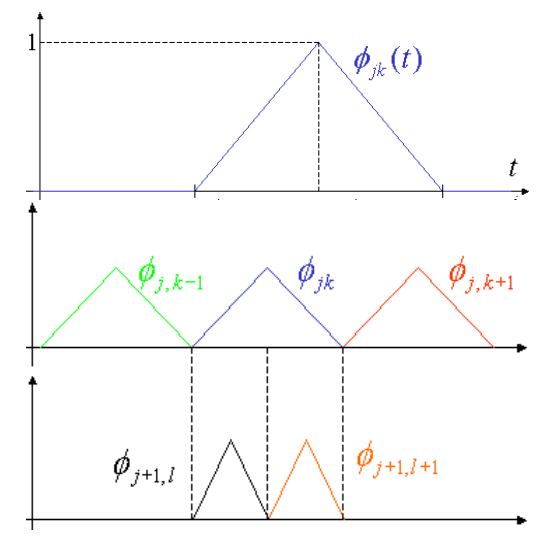

Figure 3. Triangle functions

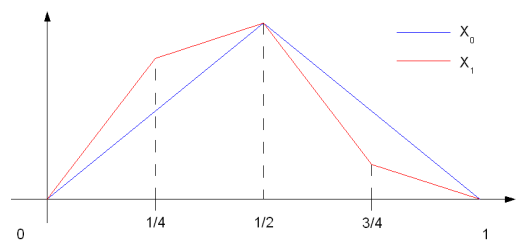

Figure 4. The processes $X_{0}$ and $X_{1}$.

Definition 3.3. Let $g$ be a $C^{1}$ function from $\mathbb{R}$ to $[a, b] \subset$ $(0,1]$. Let $Z_{j k}$ be independent and identically distributed random variables following a centered Gaussian law with unit variance. Let $X_{-1}=0$ and define the sequence $\left(X_{j}\right)_{j} \in \mathbb{N}$ recursively:

$X_{j}(t)=X_{j-1}(t)+\sum_{k=0}^{2^{j}-1} 2^{-j g\left(X_{j-1}\left(\left(k+\frac{1}{2}\right) 2^{-j}\right)\right)} Z_{j k} \varphi_{j k}(t)$.

For illustration, figure 4 shows the first stages of construction $X_{0}$ and $X_{1}$.

One can show that, almost surely, the sequence $X_{j}$ converges uniformly to a continuous process $X$ that will be termed "self-regulating midpoint displacement process" (SRMDP). The following theorem states that $X$ is indeed self-regulating:

Theorem 3.1. Almost surely, for all t:

$$
\alpha_{X}(t)=g(X(t)) .
$$

Note that $X$ is fully determined by $g$, which is called the regulating function. Some examples of SRMDP are displayed on figure 5, with an increasing $g$ function: as may be checked, the larger $X$ is, the more regular it looks.
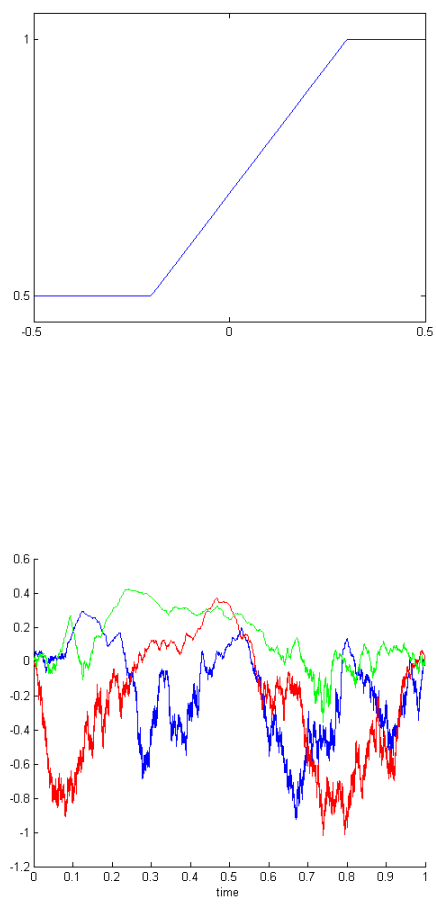

Figure 5. Top: the regulating function $g$. Bottom: three sample paths of $X$. As one can see, regions with small values look more irregular than regions with large values. 


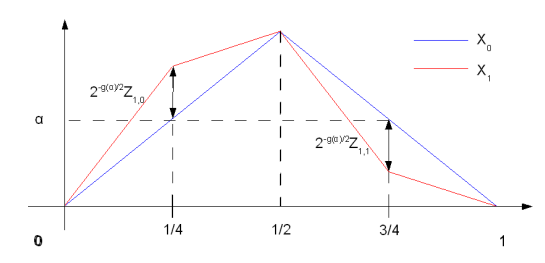

Figure 6. Information used to evaluate $g(\alpha)$.

\subsection{Estimating the self-regulating function}

Our working assumption is that a healthy heart is characterized by a typical shape of the regulating function $g$, as is suggested by figure 1 . Different types of illness, such as various kinds of arrhythmia, are likely to perturb $g$. We wish to investigate whether it is possible to measure this effect on data. Furthermore, we hope to be able to detect the effect of a treatment from the $g$ function. For these purposes, it is crucial to have at our disposal a robust method for estimating the regulating function. We briefly explain how to do so in this section. Assume we have a record of $2^{j}$ samples of $X$ (and thus $X=X_{j}$ ). We wish to estimate $g$, along with a confidence interval. Let us fist explain the method in the simplest case where $j=1$. The signal then consists of the points $X(0), X(1 / 4), X(1 / 2), X(3 / 4)$ and $X(1)$. We use these to evaluate $g$ at the point $\alpha=$ $X(1 / 2) / 2$ (see figure 6 ).

Recall that $X_{0}=Z_{0,0} \varphi_{0,0}$. Set $\alpha=\frac{1}{2} Z_{0,0}=$ $X_{0}(1 / 4)=X_{0}(3 / 4)$. Then:

$X_{1}=X_{0}+2^{-g(\alpha)} \varphi_{1,0} Z_{1,0}+2^{-g(\alpha)} \varphi_{1,1} Z_{1,1}$.

As a consequence:

$$
\begin{aligned}
& X_{1}\left(\frac{1}{4}\right)-\alpha=2^{-g(\alpha)} Z_{1,0} . \\
& X_{1}\left(\frac{3}{4}\right)-\alpha=2^{-g(\alpha)} Z_{1,1} .
\end{aligned}
$$

We thus have to estimate the common variance of two independent Gaussian random variables, which is classically done by adding their squares:

$$
\left(X_{1}\left(\frac{1}{4}\right)-\alpha\right)^{2}+\left(X_{1}\left(\frac{3}{4}\right)-\alpha\right)^{2}=2^{-2 g(\alpha)}\left(Z_{1,0}^{2}+Z_{1,1}^{2}\right) .
$$

Let $W=\left(Z_{1,0}^{2}+Z_{1,1}^{2}\right)$. $W$ follows a $\chi_{2}$ law with two degrees of freedom, and $W$ is independent of $\alpha$. Let $u, v>0$ be such that $P(W \in[u, v]) \geq 95 \%$.

Let finally:

$$
T=\left(X_{1}\left(\frac{1}{4}\right)-\alpha\right)^{2}+\left(X_{1}\left(\frac{3}{4}\right)-\alpha\right)^{2} .
$$

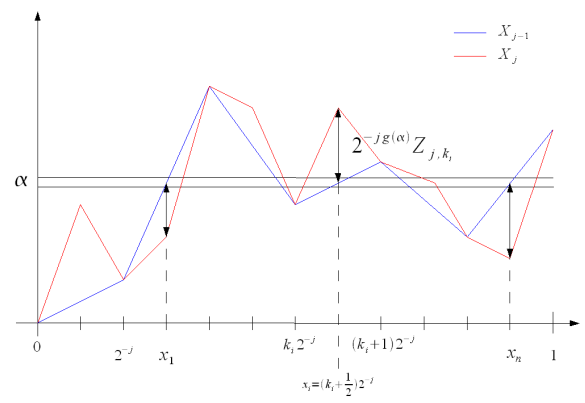

Figure 7. Processes $X_{j-1}$ and $X_{j}$.

Then, from (1):

$$
P\left(T \in\left[2^{-2 g(\alpha)} u, 2^{-2 g(\alpha)} v\right]\right) \geq 95 \%
$$

and:

$$
P\left(2^{-2 g(\alpha)} \in\left[\frac{1}{v} T, \frac{1}{u} T\right]\right) \geq 95 \%
$$

which finally yields:

$P\left(g(\alpha) \in\left[\frac{\log _{2}(u)}{2}-\frac{\log _{2}(T)}{2}, \frac{\log _{2}(v)}{2}-\frac{\log _{2}(T)}{2}\right]\right) \geq 95 \%$.

In the general case of an arbitrary $j$, fix a "small" interval $I=[\alpha-\epsilon, \alpha+\epsilon]$. Since $g$ is smooth, it is approximately constant on this interval: $g(t)=g(\alpha)$ for all $t \in I$.

Denote $x_{1} \cdots x_{n}$ the points of the form $(k+1 / 2) 2^{-j}$ such that $X_{j-1}\left(x_{i}\right) \in I$.

Let $k_{1} \ldots k_{n}$ be the integers $k$ such that $x_{i}=\left(k_{i}+\right.$ $1 / 2) 2^{-j}$. Remark that the number $n$ of points in $I$ and the integers $k_{1} \ldots k_{n}$ depend only on $X_{j-1}$, i.e. of the $Z_{l, k}, l \leq$ $j-1$. Using the definition of $X_{j}$, one gets for all $i \in[1 . . n]$ :

$$
X_{j}\left(x_{i}\right)-X_{j-1}\left(x_{i}\right)=2^{-j g(\alpha)} Z_{j k_{i}} .
$$

The shape of $X_{j-1}$ et $X_{j}$ is illustrated on figure 7 . As in the case $j=1$, one writes:

$$
\sum_{i=1}^{n}\left(X_{j}\left(x_{i}\right)-X_{j-1}\left(x_{i}\right)\right)^{2}=2^{-2 j g(\alpha)} \sum_{i=1}^{n} Z_{j k_{i}}^{2} .
$$

Let $W=\sum_{i=1}^{n} Z_{j k_{i}}^{2}$. Conditionally on $n, W$ follows a $\chi_{2}$ law with $n$ degrees of freedom. Let $[u(n), v(n)]$ be a confidence interval at $95 \%$ for a $\chi_{2}$ law with $n$ degrees of freedom, $u(n), v(n)>0$. Then, for all $n$ :

$P(W \in[u(n), v(n)] \mid n) \geq 95 \%$. from (2):

Let $T=\sum_{i=1}^{n}\left(X_{j}\left(x_{i}\right)-X_{j-1}\left(x_{i}\right)\right)^{2}$. Then,

$$
P\left(T \in\left[2^{-2 g(\alpha)} u(n), 2^{-2 g(\alpha)} v(n)\right]\right) \geq 95 \%
$$




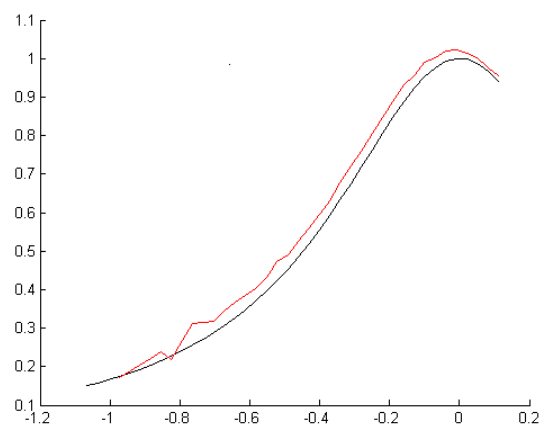

Figure 8. True (black) and estimated (red) $g$ for an SRMDP.

and finally:

$P\left(g(\alpha) \in\left[\frac{\log _{2}(u(n))}{2}-\frac{\log _{2}(T)}{2}, \frac{\log _{2}(v(n))}{2}-\frac{\log _{2}(T)}{2}\right]\right) \geq 95 \%$.

We display on figure 8 the result of estimating $g$ on an SRMDP (not shown) sampled on 65436 points. As one can see, the fit is quite good.

Figure 9 displays a path of $X$ with regulating function $g(z)=\frac{1}{1+5 z^{2}}$, along with confidence intervals at level $96 \%$ computed with two values of $\epsilon$.

\section{Application to arrhythmia analysis}

Several articles have shown that (multi-)fractal parameters are able to discriminate healthy subjects from patients suffering from various kinds of arrhythmia, see e.g. [9], or to distinguish between ventricular fibrillation and tachycardia [2]. Our work further supports this by showing that a fine analysis of the time evolution of regularity (as opposed to a global analysis of the distribution of regularity, as is multifractal analysis) allows to discriminate healthy hearts from ones suffering from arrhythmia, but also to assess the efficiency of certain treatments. We mention here that only few papers deal with measuring the effect of drugs for restoring the multifractal properties of heart rate variability. Let us mention [1] that shows that cardivelol treatment restored multifractality in patients with congestive heart failure.

We shall present results on the following database:

- 78 RR records of healthy subjects,

- $760 \mathrm{RR}$ records of patients suffering from ventricular extrasystole,

- 270 RR records from the 760 patients above which were subsequently treated with encainide,
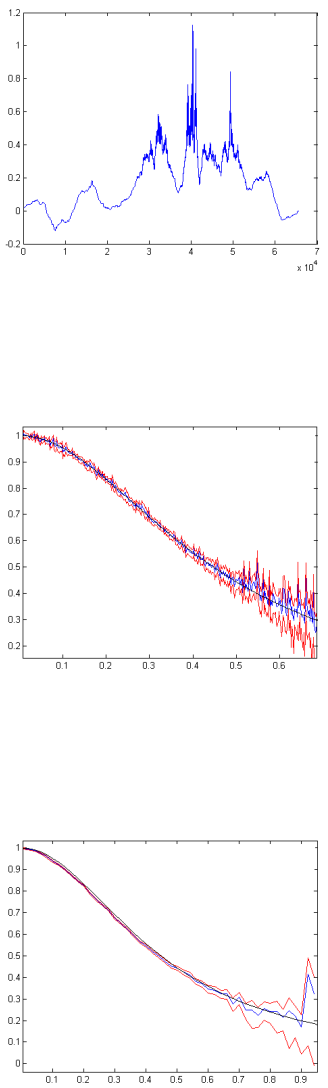

Figure 9. SRMDP with 65436 points (top), $96 \%$ Confidence intervals with $\epsilon=0.002$ (middle), $96 \%$ Confidence intervals with $\epsilon=0.02$ (bottom). 
- 215 RR records from the 760 patients above which were subsequently treated with flecainide,

- 275 RR records from the 760 patients above which were subsequently treated with moracizine.

All our RR signals are 24 hours long.

Ventricular extrasystole is the most common type of arrhythmia. It is caused by a premature ventricular depolarization originating between the Tawara node and the His fascicle. It may occur either as a result of at risk behaviors, such as excessive consumption of coffee or tobacco, or may be caused by coronary artery disease, cardiomyopathy or other conditions. In terms of ECG, it translates into an lengthened QRS complex, with typically vanishing of the $\mathrm{P}$ wave. Such a modification has an impact on the RR signal, which should be noticeable on the regularity function: indeed, longer beats will entail sudden discontinuities in the signal, which in turn will result in an overall decrease of the Hölder exponents at points close to the extrasystoles. When $g$ is evaluated at the values taken by $X$ at these points, these smaller exponents will contribute to diminish the value of $g$. Now, the values of $X$ corresponding to ventricular extrasystole are the larger ones, because these episodes precisely translate into longer intervals between two "R". We thus expect the regularity function for $\mathrm{RR}$ records with arrhythmia to be lower as compared to healthy RR, specially where the RR signal is large. This means that $g(x)$ for "large" $x$ should be smaller in the case of arrhythmia.

We studied the database described above in order to:

1. check whether healthy RR are indeed self-regulating: this means that a $g$ function may be satisfactorily estimated from the data,

2. check whether one can discriminate healthy RR records from ones with arrhythmia through the regulating function,

3. check whether one can assess the efficiency of the three treatments above, again through an analysis of the regulating function.

\subsection{Healthy RR intervals are self-regulating}

We verify the self-regulating assumption with two approaches. First, for a given patient, we estimate the $g$ function along with the confidence interval. A small interval means that the regulating is well defined, in other words that the RR signal is indeed self-regulating. We recall here that a point $(x, g(x))$ in the graph of $g$ heuristically means that an interbeat of duration $x$ has regularity $g(x)$.

We show on figure 10 a typical $g$ function with its confidence interval on a healthy RR signal. As one can check, the estimation is extremely precise. The decreasing overall shape of $g$ is consistent with figure1, which showed that longer interbeats (and thus points $x$ in the graph of $g$

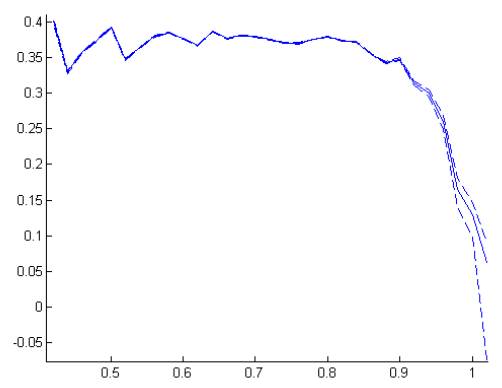

Figure 10. Typical $g$ function with confidence interval for a healthy RR.

with larger abscissa) are typically less regular (i.e have a smaller $g(x))$ than shorter ones.

The second test is a statistical one: we estimate the $g$ function for all healthy RR records. As one can see it on figure 11, most regulating functions look the same. This means two things: first, there is indeed some relevant information in the $g$ function. Second, for most healthy hearts, the self-regulation is roughly the same. In other words, the information captured by $g$ is robust across a healthy population.

\subsection{Discriminating healthy $R R$ intervals from ones with arrhythmia}

On figure 11, one can see that almost all $g$ functions lie above the line with equation $y=0.48-0.24 x$, displayed in green. According to our analysis above, we expect that arrhythmia due to ventricular extrasystole will decrease $g$. More precisely, ventricular arrhythmia entails longer heartbeats, which are more irregular. Thus, we specifically expect the values of $g(x)$ for large $x$ to be smaller here than in the healthy case.

This fact may be verified on figure 12 , where are displayed the $g$ functions of all the patients with arrhythmia. There are three such graphics, because the curves are grouped by sets of patients that will subsequently by treated with the same drug, i.e. encainide, flecainide or moracizine. At this stage, however, no treatment has been administered.

The regulating functions appear chaotic, with no apparent common pattern, and do not stay above the line $y=0.48-0.24 x$. For a more quantitative statement, see table 1, where the proportions of curves which are above this line are given for all the populations. 


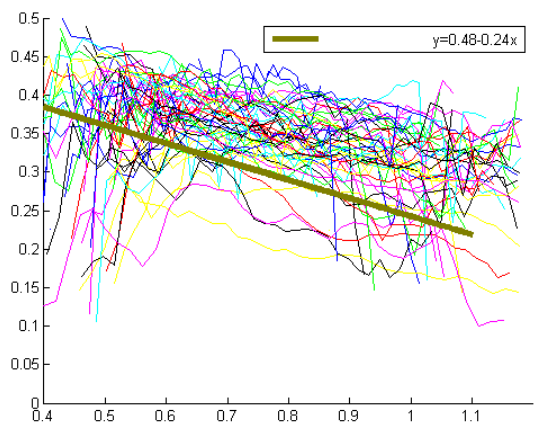

Figure 11. Estimations of $g$ on all healthy RR.

$\begin{array}{ll}\text { Healthy RR } & 10 \% \\ \text { Group E before treatment } & 86 \% \\ \text { Groupe E after treatment } & 50 \% \\ \text { Groupe F before treatment } & 80 \% \\ \text { Groupe F after treatment } & 28 \% \\ \text { Groupe M before treatment } & 90 \% \\ \text { Groupe M after treatment } & 46 \%\end{array}$

Table 1. Proportion of signals with $g$ function below the line $y=0.48-0.24 x$ for healthy $\mathrm{RR}$, and groups prior and after treatment with encainide (E), flecainide (F) and moracizine (M).

\subsection{Assessing the effect of treatments}

Figure 13 displays the regulating functions for the patients in the three groups during treatment. Overall, we can see that the shape of $g$ is affected by the treatment, to the effect that it increases in average, in particular for larger values of the amplitude (i.e. to the right of the graphs): this exactly means that longer heartbeats, which are comparatively regular for healthy RR and irregular for arrhythmic $\mathrm{RR}$, become again noticeably more regular after medication. These longer intervals are where ventricular extrasystoles manifest themselves. This suggest that the three drugs do have an effect on the targeted pathology.

\subsection{Statistical analysis}

To give a more quantitative content to our findings, we provide in Table 1 the statistics of the number of RR records which are below the line $y=0.48-0.24 x$ in all groups.

One sees that this simple criterion allows to discriminate with a good precision healthy RR from ones with arrhythmia. Also, the three treatments seem to improve the

\begin{tabular}{|cc|ccc|ccc|ccc|}
$\mathrm{A}$ & $\mathrm{H}$ & $\mathrm{NH}$ & $\mathrm{E}$ & $\mathrm{H}$ & $\mathrm{NH}$ & $\mathrm{F}$ & $\mathrm{H}$ & $\mathrm{NH}$ & $\mathrm{M}$ & $\mathrm{H}$ \\
\hline 98 & 89 & 13 & 96 & 4 & 1 & 90 & 7 & 1 & 93 & 5
\end{tabular}

Table 2. Pourcentage of well-classified signals. $A=a r-$ rhythmic, $\mathrm{H}=$ healthy, $\mathrm{E}=$ treated with encainide, $\mathrm{F}=$ treated with flecainide, $\mathrm{M}=$ treated with moracizine.

situation, with a greater efficiency for flecainide (the fact moracizine seems less effective than flecainide is in agreement with other studies in the literature).

We finally show results on the supervised discrimination of all groups based on the regulating function, with a classical linear method implemented in MatLab. We have used two thirds of the data for the learning phase, and the remaining for the test. As can be seen on Table 2, when considering only the two populations "healthy" and "arrhythmic", the results of classification are excellent. However, as soon as one introduces a new class, i.e. a group of patients treated either by encainide, flecainide or moracizine, the quality drops dramatically. This indicates that RR records of treated patients do not belong to a well defined class. A more refined classification of the $g$ functions of the treated patients (not displayed here) allows to quantitatively assess the efficiency of the three drugs by computing the proportions of patients which have recovered a "normal" regulating function.

\section{Conclusion}

We have proposed a new model for RR intervals that takes into account the fact heartbeats are not only extremely variable (multifractal), but also self-regulating. We have designed a statistical estimator of the self-regulating function. Applying this to RR records, we were able to discriminate clearly healthy subjects from ones suffering from ventricular extrasystole. In addition, the efficiency of three treatments has be assessed, by measuring how much the selfregulating function recovers its normal shape after medication. Further work will concentrate on a more precise analysis of the self-regulating function to check whether it may be used for refined disease characterization.

\section{References}

[1] K.M. Chiu, H.L. Chan, S.H. Chu, and T.Y. Lin. Carvedilol can restore the multifractal properties of heart beat dynamics in patients with advanced congestive heart failure. Auton Neurosci., 132:76-80, 2007.

[2] Z. Daoming, T. Guojun, H. Jifei, and H. Yaofei. Ventricular arrhythmia nonlinear analysis. In IPC '07: Proceedings of the The 2007 International Conference on Intelligent Pervasive Computing, pages 57- 

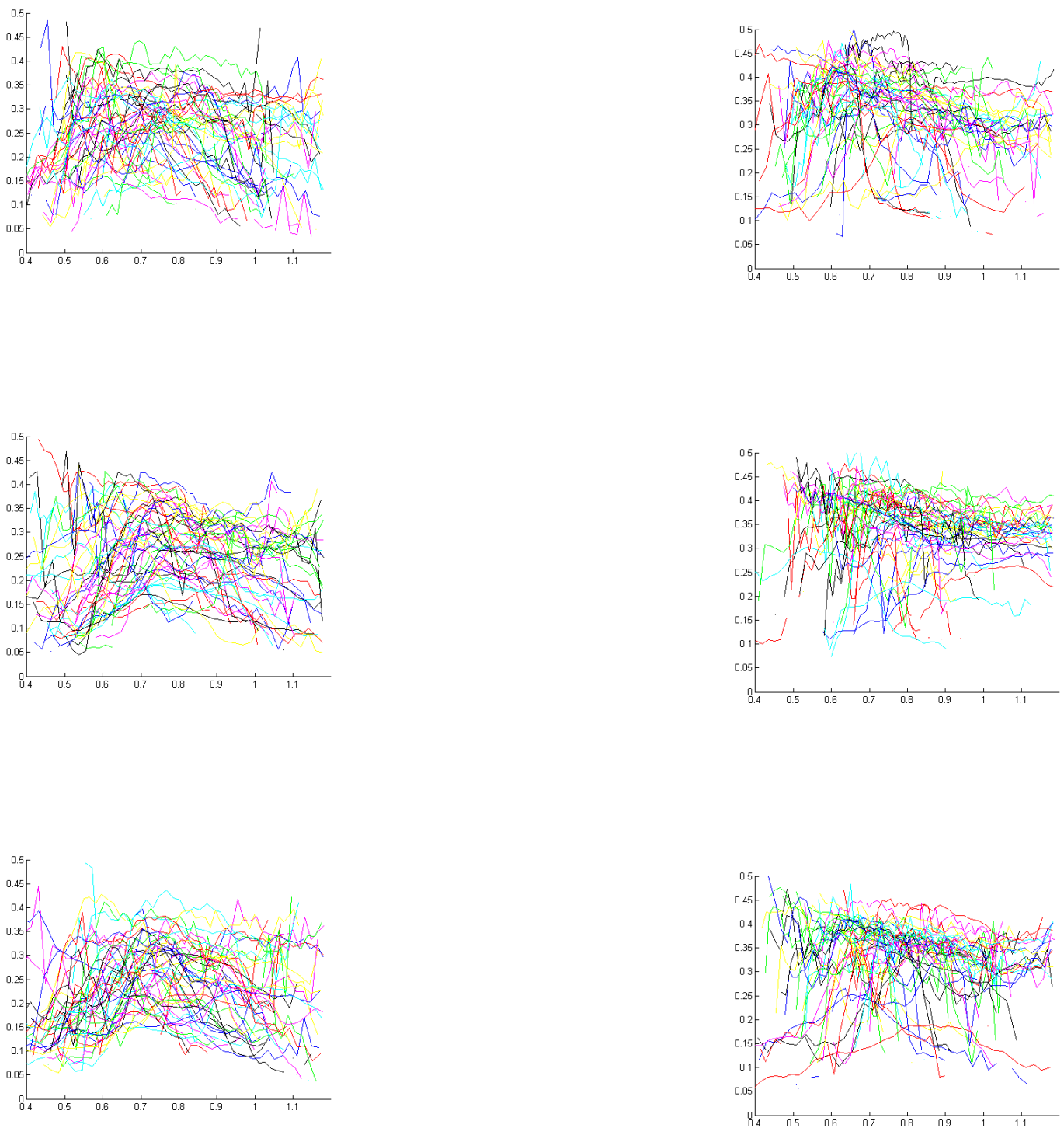

Figure 12. Regulating functions for RR with arrhythmia grouped by patients that will be subsequently treated with encainide (top), flecainide (middle) and moracizine (bottom).
Figure 13. Regulating functions for RR with arrhythmiatreated with encainide (top), flecainide (middle) and moracizine (bottom). 
61, Washington, DC, USA, 2007. IEEE Computer Society.

[3] A. Echelard, O. Barrière, and J. Lévy Véhel. Terrain modelling with multifractional brownian motion and self-regulating processes. In ICCVG 2010, volume 6374 of Lecture Notes in Computer Science, pages 342-351, Warsaw, Poland, 2010. Springer.

[4] S. Ezekiel and A.A. Alshehri. Heart rate variability signal processing by using wavelet based multifractal analysis. In Digital Signal Processing and Control, IASTED International Conference, Measurement and Control (MECO'2001), 2001.

[5] R. Fischer, M. Akay, P. Castiglioni, and M. Di Rienzo. Multi-and monofractal indices of short-term heart rate variability. Medical and Biological Engineering and Computing, 41(5):543-549, 2003.

[6] P.C. Ivanov, L.A. Nunes Amaral, A.L. Goldberger, S. Havlin, M.G. Rosenblum, Z.R. Struzik, and H.E. Stanley. Multifractality in human heartbeat dynamics. Nature, 399:461-465, 1999.

[7] M. Meyer and O. Stiedl. Fractal rigidity by enhanced sympatho-vagal antagonism in heartbeat interval dynamics elicited by central application of corticotropin-releasing factor in mice. J Math Biol., 22:830-874, 2006.

[8] Y. Meyer, O. Stiedl, and B. Kerman. Discrimination by multifractal spectrum estimation of human heartbeat interval dynamics. Fractals-Complex Geometry Patterns and Scaling in Nature and Society, pages 195-204, 2003.

[9] C. Raab, N. Wessel, A. Schirdewan, and J. Kurths. Large-scale dimension densities for heart rate variability analysis. Physical Review E, 73, 2006.

[10] F. Roueff and J. Lévy-Véhel. A regularization approach to fractional dimension estimation. In Proceedings of Fractals 98, Malta, October, 1998.

[11] R. Sassi, M.G. Signorini, and S. Cerutti. Multifractality and heart rate variability. Chaos, 19:028507, 2009.

[12] G. Wang, H. Huang, H. Xie, Z. Wang, and X. Hu. Multifractal analysis of ventricular fibrillation and ventricular tachycardia. Med Eng Phys., 29:375-379, 2007.

[13] Y. Wang, Y.S. Zhu, N.V. Thakor, and Y.H. Xu. A short-time multifractal approach for arrhythmia detection based on fuzzy neural network. IEEE Trans Biomed Eng., 48:989-995, 2001. 